\title{
Infective endocarditis complicated by aortic graft infection and osteomyelitis: case report and review of literature
}

This article was published in the following Dove Press journal:

International Journal of General Medicine

4 July 2012

Number of times this article has been viewed

\author{
Elie Zouein' \\ Robert Wetz' \\ Neville Mobarakai' \\ Samer Hassan' \\ Iris Tong ${ }^{2}$ \\ 'Department of Medicine, Staten \\ Island University Hospital, New York, \\ NY, USA; ${ }^{2}$ Department of Medicine, \\ Warren Alpert Medical School of \\ Brown University, Providence, RI, USA
}

Correspondence: Elie Zouein Staten Island University Hospital 475 Seaview Ave, Staten Island, New York, NY, USA Email ezouein@siuh.edu/lalous66@ hotmail.com

\begin{abstract}
Primary aortic graft infection early after aortic graft insertion is well described in the literature. Here, we present a unique case of late aortic graft infection 5 years after insertion secondary to mitral valve endocarditis, resulting from cellulitis in a patient with severe venous varicosities. A 63-year-old male presented for severe low back pain, constipation, and low-grade fever. An abdominal computed tomography scan with oral and intravenous contrast showed a normal spine and urinary tract. Blood and urine cultures, done at the same time, grew Staphylococcus aureus. A transesophageal echocardiogram confirmed the diagnosis of endocarditis. Subsequently, a gallium scan showed increased uptake in the vertebral bodies, aortic graft, left patella, and left ankle. After 3 months of antibiotic therapy, the patient's low back pain resolved with normalization of his laboratory values. He remained free of infection at a 2-year follow-up. We reviewed the literature concerning the atypical presentation of infective endocarditis, with a focus on distant metastases at initial presentation, such as osteomyelitis and aortic graft infection, as well as the different treatment modalities. This report describes successful medical treatment with intravenous followed by oral antibiotics for an infected endovascular graft without any surgical intervention.
\end{abstract}

Keywords: endocarditis, osteomyelitis, aortic graft infection, septic emboli, endovascular abdominal aortic aneurysm repair (EVAR)

\section{Case report}

A 63-year-old male presented with severe low back pain and constipation. The patient had been doing well until 5 days before presentation when he experienced acute-onset lumbar back pain. The pain was described on a scale of 10 as being 9, was sharp, throbbing, worse with movement, and without radiation or alleviating factors. Four milligrams of intravenous (IV) morphine, given in the emergency room, did not provide any pain relief. The patient had not had a bowel movement in the previous 10 days. He denied any recent trauma, fever, headache, weight loss, cough, hemoptysis, or urinary/stool incontinence.

The patient had a history of lumbago, chronic obstructive pulmonary disease, coronary artery disease, hypertension, peripheral vascular disease (postendovascular abdominal aortic aneurysm repair 5 years prior), dyslipidemia, diabetes mellitus, diabetic neuropathy, lower extremity varicose veins (postsurgical stripping), benign prostatic hypertrophy, schizophrenia, anxiety, and depression. He was an active smoker (10 cigarettes per day) for many years. He denied alcohol or drug use. He had a positive family history of cancer and myocardial infarction. He had no known medication or food allergies. 
On arrival, his temperature was $99.6^{\circ} \mathrm{F}$, blood pressure was 124/81 $\mathrm{mmHg}$, pulse was $84 \mathrm{bpm}$, respiratory rate was 20 per minute, and oxygen saturation was $97 \%$ on room air. The patient was in obvious discomfort, noticeably sweating and grunting secondary to the pain. There was no jugular venous distension or lymphadenopathy. Chest examination revealed decreased breath sounds bilaterally without crackles or wheezes. Heart sounds were regular without murmurs, gallops, or rubs. His abdomen had positive bowel sounds, and was obese, soft, nontender, and nondistended. Rectal exam showed good sphincter tone and no fecal impaction. There was trace edema in the lower extremities with severe varicosities, greater on the left side, with intact skin. Femoral, popliteal, and pedal pulses were normal on both sides. Musculoskeletal exam demonstrated mild to moderate tenderness in the midspine and left lumbar areas, superior to the iliac crest.

On presentation, laboratory data showed a white blood cell (WBC) count of $10^{*} 10^{12} / \mathrm{L}$, with $88 \%$ neutrophils, $13 \mathrm{~g} / \mathrm{dL}$ hemoglobin, and $150 * 10^{12} / \mathrm{L}$ platelets. His blood urea nitrogen and creatinine were 29 and $1.11 \mathrm{mg} / \mathrm{dL}$, respectively. Sodium was $130 \mathrm{mmol} / \mathrm{L}$ and potassium was $4.6 \mathrm{mmol} / \mathrm{L}$. Cardiac, liver, and pancreatic enzymes were within normal limits. Electrocardiogram showed normal sinus rhythm at $82 \mathrm{bpm}$ with right-bundle branch block and left anterior hemiblock. Chest radiograph was unremarkable. Computed tomography (CT) scan of the abdomen and pelvis with IV and oral contrast was performed and showed a large amount of stool in the colon without bowel obstruction or perforation. There was no free intra-abdominal air or fluid collection. The aortobiiliac graft was patent without perigraft fluid (Figure 1A and B).

The patient was admitted for severe back pain and constipation. He had no fever at the time of admission. A repeat complete blood count (CBC) showed a white count of $13 * 10^{12} / \mathrm{L}$. The erythrocyte sedimentation rate (ESR) was $52 \mathrm{~mm} / \mathrm{h}$. Later on, he developed a temperature of $100.6^{\circ} \mathrm{F}$. Blood cultures were drawn.

On hospital day 2, he had an episode of severe shortness of breath. CT angiography of the chest was negative for pulmonary embolism (PE). Urine analysis and urine culture as well as repeat blood cultures were obtained.

On hospital day 3, the first blood culture grew Gram-positive cocci in clusters, which were later identified as oxacillin-sensitive Staphylococcus aureus (OSSA). The patient continued to have low-grade temperatures and the second blood culture and urine culture also grew OSSA. S. aureus seemed an unlikely pathogen for a primary urinary tract infection, and thus a search for endocarditis ensued. Ciprofloxacin had been started, but this was changed to

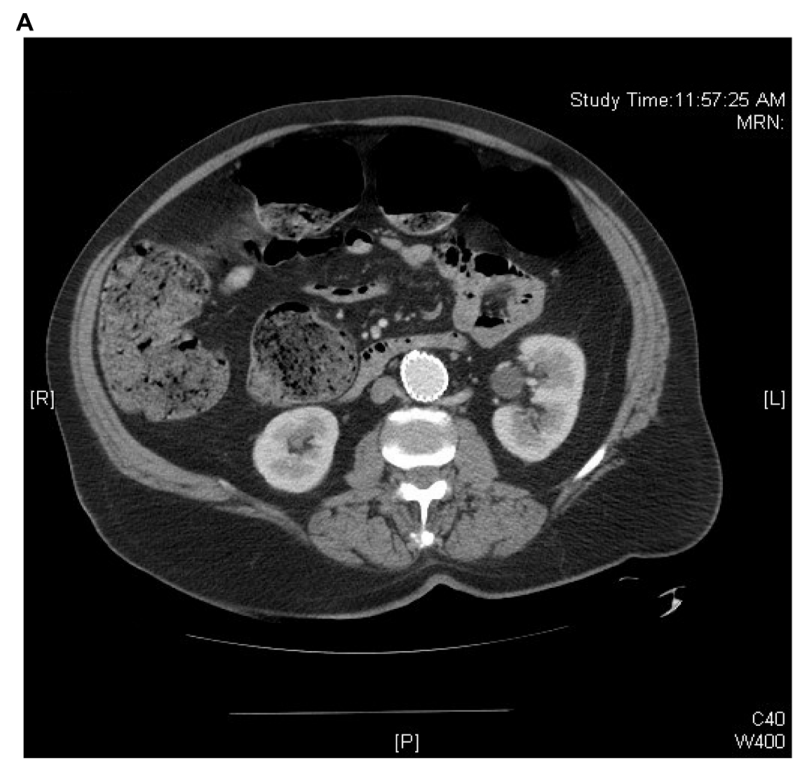

B



Figure I Computed tomography scans showing the endovascular aortic graft with no evidence of free intra-abdominal air or fluid collection.

Notes: (A) Patent endovascular aortic graft at the level before the iliac bifurcation. (B) Patent endovascular aortic graft at the level of iliac bifurcation. In both images there is no evidence of free intra-abdominal air or fluid collection.

oxacillin following the culture results. A repeat ESR was $54 \mathrm{~mm} / \mathrm{h}$. The WBC count rose to $18^{*} 10^{12} / \mathrm{L}$. A transthoracic echocardiogram was negative, but a subsequent transesophageal echocardiogram (TEE) showed a small mobile vegetation on the tip of the anterior leaflet of the mitral valve, confirming the diagnosis of bacterial endocarditis. Meanwhile, the patient continued to complain of low back pain. On hospital day 7, a third blood culture was again positive. The previous $\mathrm{CT}$ scan of the abdomen and pelvis was reviewed with the radiologist and no spinal involvement was found. Subsequently, a fourth blood culture was also positive for OSSA. Gentamicin and rifampin 
were added for the possibility of an aortic endovascular graft infection. A gallium scan was performed and showed increased uptake in the thoracic vertebral bodies T11 and T12, the aortic graft, left patella, and left ankle (Figures 2 and 3 ). Over the following days, the WBC count decreased to $14 * 10^{12} / \mathrm{L}$; a fifth blood culture drawn on day 12,9 days after initiation of oxacillin, was negative.

This patient's source of endocarditis was probably the lower extremities; later, an open wound on the left ankle was discovered, which had not been present at the time of admission. This probably seeded the blood stream and then the mitral valve, with subsequent septic emboli to the vertebral bodies, left knee, patella, and aortic graft. The vascular surgery team decided that no intervention was warranted for the endovascular graft and recommended to continue IV antibiotics and provide local wound care to the left ankle. A sixth blood culture was negative.

Swelling, redness, and pain decreased and the patient's leg healed rapidly. Repeated CBC, ESR, and C-reactive protein (CRP) tests showed a normalization of the patient's WBC and increases in ESR to $83 \mathrm{~mm} / \mathrm{h}$ and CRP to $9.9 \mathrm{mg} / \mathrm{dL}$. The patient was discharged to a nursing home. The antibiotics were narrowed to oral rifampin and IV cefazolin and gentamicin, which were continued for 6 weeks after the first negative blood culture. ESR, CRP, CBC, and a basic metabolic panel were checked every 2 weeks. At 6 weeks follow-up, the patient's ESR was $<35 \mathrm{~mm} / \mathrm{h}$ and CRP $<3 \mathrm{mg} / \mathrm{dL}$. Hence the antibiotics were changed to oral dicloxacillin $500 \mathrm{mg}$ every 6 hours for 2 additional months. The patient was discharged from the nursing home and instructed to continue the same wound care of his left leg until the skin was fully intact.

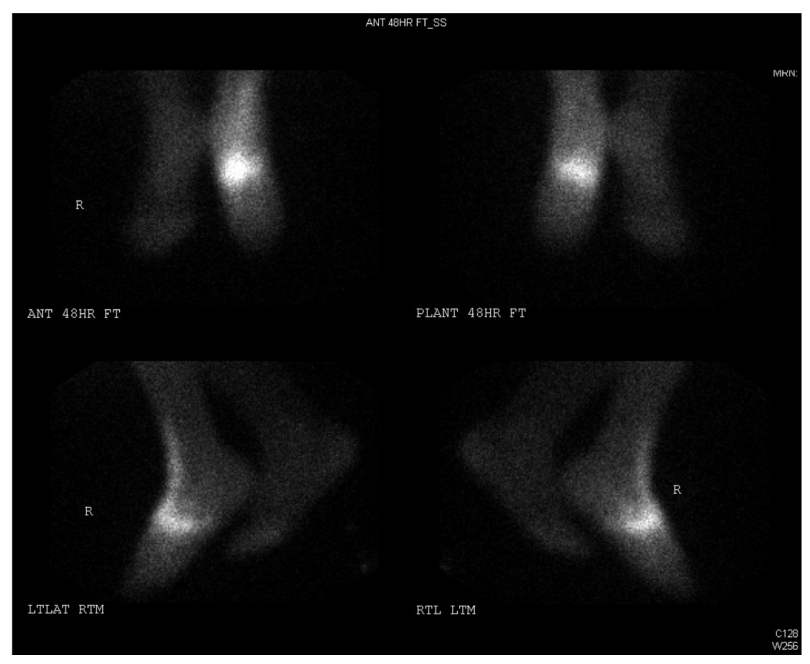

Figure 2 Gallium scan of the feet. Intense gallium uptake traversing the left ankle most intense medially, consistent with inflammatory joint abnormality.

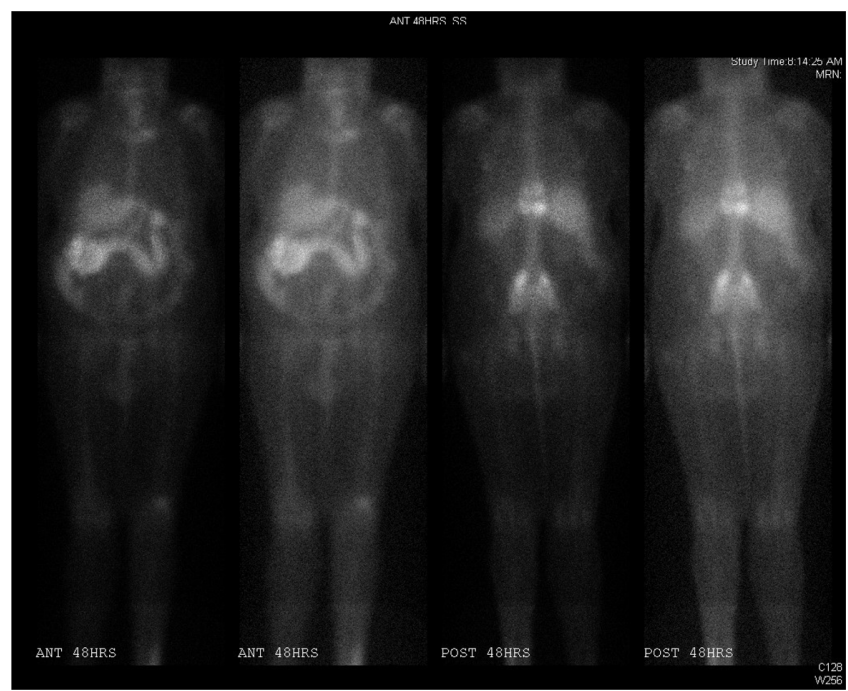

Figure 3 Whole body gallium scan.

Notes: The first 2 images from the left show increased uptake in the left knee. The remaining 2 images show abnormal gallium uptake on the right side of the vertebral body and pedicle of a lower thoracic vertebra, with increased uptake in the vertebra immediately superior consistent with osteomyelitis/diskitis. Abnormal gallium uptake on both sides of the midline in the lumbar region, representing uptake in the right and left iliac components of the endovascular stent, consistent with an active inflammatory process.

His ESR decreased to $27 \mathrm{~mm} / \mathrm{h}$ and his CRP normalized. These results were unchanged 2 weeks after stopping all antibiotics and a repeat blood culture was also negative. At a 2-year follow-up, the patient remained free of infection.

\section{Discussion}

\section{Infective endocarditis (IE): diagnosis, epidemiology and microbiology}

Definite diagnosis of IE relies on using the Duke criteria, which include two major criteria or one major and three minor criteria (Table 1).

Bayer et al found that $26 \%$ of IE patients present without a history of drug addiction or previous valvular lesion. They also found that $77 \%$ of patients with definite IE had bacteremia and $57 \%$ had vegetations, of which $41 \%$ were only seen by TEE. ${ }^{1}$ In another study by Heiro et al, $72 \%$ of IE patients had vegetations. In the non-IV drug-user group, the most common etiology was $S$. aureus (23\%) followed by Streptococcus viridans (17\%). ${ }^{2}$ The International Collaboration on Endocarditis Prospective Cohort Study showed that, overall, $42 \%$ of IE was staphylococcal in origin with $S$. aureus accounting for $31 \%$, viridans-group Streptococci 17\%, and Enterococci $11 \%{ }^{3}$ Echocardiography should be performed in patients where IE is highly suspected. Sensitivity of transthoracic echocardiogram in detecting vegetations is $50 \%-60 \%$ and for TEE, it approaches $90 \%$, especially for vegetations $<5 \mathrm{~mm}{ }^{4}$ The negative predictive value of TEE is nearly $100 \%$. 
Table I The Duke Criteria for the clinical diagnosis of infective endocarditis (IE)

\section{Major criteria}

I. Positive blood cultures for IE

- Typical microorganism for infective endocarditis from two separate blood cultures:

- viridans streptococci, Streptococcus bovis, HACEK group, Staphylococcus aureus, or

- Community-acquired Enterococci in the absence of a primary focus or

- Persistently positive blood culture, defined as recovery of a microorganism consistent with infective endocarditis from:

- blood cultures drawn $>12$ hours apart, or

- all of three or a majority of four or more separate blood cultures, with first and last drawn at least I hour apart

or

- Single positive blood culture for Coxiella burnetii or phase I lgG antibody titer of $>1: 800$

2. Evidence of endocardial involvement

- Positive echocardiogram:

- oscillating intracardiac mass on valve or supporting structures or in the path of regurgitant jets or in implanted material, in the absence of an alternative anatomic explanation, or

- abscess, or

- new partial dehiscence of prosthetic valve,

or

- New valvular regurgitation (increase or change in preexisting murmur not sufficient)

\section{Minor criteria}

I. Predisposition: predisposing heart condition or injection drug use

2. Fever $\geq 38.0^{\circ} \mathrm{C}\left(\geq 100.4^{\circ} \mathrm{F}\right)$

3. Vascular phenomena: major arterial emboli, septic pulmonary infarcts, mycotic aneurysm, intracranial hemorrhage, conjunctival hemorrhages, Janeway lesions

4. Immunologic phenomena: glomerulonephritis, Osler's nodes, Roth's spots, rheumatoid factor

5. Microbiologic evidence: positive blood culture but not meeting major criterion as noted previously ${ }^{\mathrm{b}}$ or serologic evidence of active infection with organism consistent with infective endocarditis

Notes: ${ }^{\text {TT }}$ ransesophageal echocardiography is recommended for assessing possible prosthetic valve endocarditis or complicated endocarditis; bexcluding single positive cultures for coagulase-negative Staphylococci and diphtheroids, which are common culture contaminants, and organisms that do not cause endocarditis frequently, such as Gram-negative bacilli.

Copyright (c) 2000, Oxford University Press. Adapted with permission from Li JS, Sexton DJ, Mick N, et al. Proposed modifications to the Duke criteria for the diagnosis of infective endocarditis. Clin Infect Dis. 2000;30(4):633-638.19

Abbreviations: HACEK, Haemophilus spp., Actinobacillus actinomycetemcomitans, Cardiobacterium hominis, Eikenella corrodens, Kingella kingae; IgG, immunoglobulin G.

\section{Infective endocarditis: manifestations} Musculoskeletal

Musculoskeletal manifestations occur frequently in IE and can be part of the initial presentation. Lumbar pain is reported in subacute IE and is thought to be related to either embolization or septic necrosis of the intervertebral space. ${ }^{4}$ Churchill et al indicated in their study that $15 \%$ of patients with IE had musculoskeletal manifestations as the presenting symptom, including arthralgia in 38\% with a predilection to proximal joints (most frequently the shoulder followed by the knee and the hip), arthritis/synovitis (with the ankle most commonly affected) in $31 \%$, lumbar pain in $23 \%$, diffuse myalgia in $19 \%$, and disc infection in $8 \%$. They also found that lumbar pain preceded the diagnosis of IE by about 1.5 months. ${ }^{5}$ Additionally, the development of acute synovitis in certain unusual locations - such as in sternoclavicular or acromioclavicular joints or a single metacarpophalangeal joint - are infrequently involved in common forms of arthritis and should alert the clinician to the possibility of IE. ${ }^{5}$

Another study by Levo and Nashif, regarding musculoskeletal manifestations of IE revealed that $25 \%$ of IE cases exhibited musculoskeletal manifestations including arthralgia $(23 \%-74 \%)$, arthritis $(22 \%-41 \%)$, myalgia $(23 \%-48 \%)$, and low back pain (up to $43 \%)$. Knees were the most commonly affected joints. There was a female preponderance $(2: 1)$ in patients presenting with musculoskeletal manifestations, which again preceded the diagnosis by several weeks. ${ }^{6}$

\section{Vertebral osteomyelitis}

Despite being a well-known complication of IE, vertebral osteomyelitis is a relatively rare complication. Osteomyelitis is believed to occur in only $2 \%-6 \%$ of cases of IE and is more common in IV drug abusers. ${ }^{7}$ The most common identifiable primary sources of this complication are the genitourinary tract (29\%), followed by soft tissue infection (13\%) and upper respiratory tract infection $(11 \%) .{ }^{7}$ Isolated osteomyelitis is caused by $S$. aureus in $60 \%-90 \%$ of cases and by Staphylococcus epidermidis in 30\%. Staphylococcus spp. are more commonly associated with embolic and suppurative complications. ${ }^{\text {? }}$

The initial diagnostic approach for vertebral osteomyelitis includes a physical examination, ESR and/or CRP, blood cultures, and plain radiographs of the painful portions of the spine. An elevated ESR or CRP with negative plain films warrants further investigation. CT scan findings such as end plate irregularities may not be specific for osteomyelitis and early changes may be missed. MRI, if not contraindicated, is the most sensitive radiologic technique to detect vertebral osteomyelitis and is superior to $\mathrm{CT}$ scanning for detection of epidural abscesses. When the radiographic findings on plain films or CT scan are equivocal and the suspicion of vertebral osteomyelitis is high, radionuclide scanning may be useful. Three different techniques can be used: bone, tagged WBC, and gallium scans. Bone scans use technetium- $99 \mathrm{~m}$ and have 
a high specificity and sensitivity, but false positive results are more likely to occur when noninfectious disorders are seen on radiographs. ${ }^{8}$ Tagged WBC scans use autologous WBCs labeled with indium-111, gallium-67, or technetium-99 m and are similar in sensitivity to bone scans for evaluation of osteomyelitis, but the specificity can be poor if corresponding radiographs are not normal. WBC scans are more useful in the distal extremities because regions with substantial quantities of red marrow, such as the vertebral bodies, are not visualized reliably with this modality. Finally, gallium scans use Ga-67 and are highly sensitive and more specific than technetium- $99 \mathrm{~m}$ scans and have a high negative predictive value. However, false positive results can occur in the settings of fracture or bone neoplasm. With a positive imaging study suggestive of vertebral osteomyelitis, a CTguided needle biopsy of the affected bone should be done, and the specimen sent for pathology and culture. A needle biopsy may not be necessary if blood cultures are positive and there are typical clinical and radiographic findings of vertebral osteomyelitis.

\section{Aortic graft infection (AGI)}

AGI can result from bacteremia secondary to embolization from a valvular vegetation. Outside the setting of IE, AGI must be suspected when the patient with an aortic graft develops unexplained fever and back or abdominal pain. ${ }^{9}$ Also highly suggestive of AGI are progressive renal failure with bilateral hydronephrosis as a result of retroperitoneal inflammation, a positive arterial blood culture distal to the graft, or detection of periaortic inflammation on gallium scan. ${ }^{9}$

The diagnostic approach for AGI uses nearly the same parameters as those used for vertebral osteomyelitis. The initial diagnostic modality of choice is the $\mathrm{CT}$ scan, which has a sensitivity and specificity of $94 \%$ and $85 \%$, respectively. ${ }^{10}$ CT scan findings include perigraft fluid, perigraft soft tissue attenuation, ectopic gas, pseudoaneurysm, focal bowel wall thickening, and hydronephrosis..$^{10}$ Magnetic resonance imaging has comparable sensitivity and specificity, and helps to differentiate between perigraft fluid collections with inflammatory changes and chronic hematomas. When $\mathrm{CT}$ readings are equivocal or have low probability, nuclear medicine scans are useful. Gallium-67 and indium-111 scans are most commonly used, with a sensitivity and specificity for the latter approaching $88 \%-100 \%$ and $50 \%-85 \%$, respectively. ${ }^{10} \mathrm{New}$ emerging investigational studies about the use of fluorodeoxyglucose positron emission tomography (FDG-PET) and FDG-PET/CT in the diagnosis of AGI are promising, ${ }^{11,12}$ with a sensitivity and specificity reaching up to $91 \%$ and $95 \%$ respectively. ${ }^{11}$ However, its efficacy was not compared with radionuclide imaging, and the technique itself was not well studied in diabetic patients in which the distribution of FDG uptake can be affected. PET is also significantly more expensive than CT and not readily available in all institutions.

\section{Infective endocarditis: treatment}

Empiric treatment for IE should be started only after two sets of blood culture are drawn. In general, antibiotic treatment should cover Staphylococci (methicillin sensitive and resistant), Streptococci and Enterococci. The antibiotics used should be bactericidal and tailored to the susceptibilities of the organism. Many studies recommend IV antibiotics for 6 weeks, while other studies report continuation with oral antibiotics after the IV course for 3-6 months, especially for more invasive organisms such as $S$. aureus. ${ }^{10-13}$ Duration of therapy may also be guided by markers such as ESR and CRP, in addition to imaging. ${ }^{10}$

In the case of AGI, the gold standard for treatment remains the explantation of the graft. For high-risk patients in whom conventional management is indicated due to severe comorbidities and who cannot tolerate surgery, complete or partial graft preservation (in case of open repair) is an option. ${ }^{10-13}$ If conservative management is the best option for the patient, it is fundamental to obtain multiple blood cultures as well as imaging studies to document the degree and resolution of the infection. Calligaro et al have suggested criteria for aortic graft preservation: the graft should be patent, the anastomoses intact, the onset of infection within 2 months of surgery, the absence of systemic infection, and the absence of Pseudomonas as a causative agent. ${ }^{14}$ Alternatively, in a retrospective review comparing surgery with percutaneous drainage before surgery, Bélair et al found that surgery after drainage appears to be safer than surgery alone. ${ }^{15}$ Other authors advocate the use of local antibiotics by instillation or irrigation of the graft. ${ }^{16,17}$ However, much of the data is derived from small studies and/or uncontrolled trials. ${ }^{15-17}$ In Numan et al's recent review of management of endograft infections, six cases in the literature with endovascular abdominal aortic aneurysm repair were managed conservatively using antimicrobial therapy and/or effusion drainage, with a mortality rate reaching up to $67 \% .{ }^{18}$ Complete removal of the stent graft followed by reconstruction or bypass is still the most effective treatment. ${ }^{18}$

\section{Conclusion}

In the case described, a conservative approach was undertaken because OSSA was the pathogen. The patient was 
given bactericidal antibiotics (gentamicin, cefazolin, and rifampicin) administered intravenously for 6 weeks after the first negative blood culture. When the ESR and CRP reached levels $<35 \mathrm{~mm} / \mathrm{h}$ and $3 \mathrm{mg} / \mathrm{dL}$, respectively, oral dicloxacillin was administered for an additional 2 months. After all antibiotics were discontinued, ESR, CRP, and blood cultures were normal.

This case demonstrates that conservative treatment can be effective in the management of AGI secondary to infective endocarditis. Although the gold standard of therapy is still surgical, there is growing evidence of successful medical management. However, further studies are still needed to confirm the likelihood of success for conservative treatment alone, especially for AGI that occurs years following implantation.

\section{Disclosure}

The authors report no conflicts of interest in this work.

\section{References}

1. Bayer AS, Ward JI, Ginzton LE, Shapiro SM. Evaluation of new clinical criteria for the diagnosis of infective endocarditis. Am J Med. 1994;96(3):211-219.

2. Heiro M, Nikoskelainen J, Hartiala JJ, Saraste MK, Kotilainen PM. Diagnosis of infective endocarditis: sensitivity of the Duke vs von Reyn criteria. Arch Intern Med. 1998;158(1):18-24.

3. Murdoch DR, Corey GR, Hoen B, et al; International Collaboration on Endocarditis-Prospective Cohort Study (ICE-PCS) Investigators. Clinical presentation, etiology, and outcome of infective endocarditis in the 21st century: the International Collaboration on Endocarditis-Prospective Cohort Study. Arch Intern Med. 2009;169(5):463-473.

4. Rangel EB, Atallah AN. Musculoskeletal manifestations of bacterial endocarditis. Sao Paulo Med J. 2000;118(5):158-160.
5. Churchill MA Jr, Geraci JE, Hunder GG. Musculoskeletal manifestations of bacterial endocarditis. Ann Intern Med. 1977;87(6):754-759.

6. Levo Y, Nashif M. Musculoskeletal manifestations of bacterial endocarditis. Clin Exp Rheumatol. 1983;1(1):49-52.

7. Lee KC, Tsai YT, Lin CY, Tsai CS. Vertebral osteomyelitis combined streptococcal viridans endocarditis. Eur J Cardiothorac Surg. 2003;23(1):125-127.

8. Buchman AL. Streptococcus viridans osteomyelitis with endocarditis presenting as acute onset lower back pain. J Emerg Med. 1990;8(3):291-294.

9. Bernhard VM. Management of graft infections following abdominal aortic aneurysm replacement. World J Surg. 1980;4(6):679-686.

10. Perera GB, Fujitani RM, Kubaska SM. Aortic graft infection: update on management and treatment options. Vasc Endovascular Surg. 2006;40(1):1-10.

11. Fukuchi K, Ishida Y, Higashi M, et al. Detection of aortic graft infection by fluorodeoxyglucose positron emission tomography: comparison with computed tomographic findings. J Vasc Surg. 2005;42:919-925.

12. Keidar Z, Engel A, Hoffman A, Israel O, Nitecki S. Prosthetic vascular graft infection: the role of ${ }^{18} \mathrm{~F}-\mathrm{FDG}$ PET/CT. $J$ Nucl Med. 2007;48(8):1230-1236.

13. FitzGerald SF, Kelly C, Humphreys H. Diagnosis and treatment of prosthetic aortic graft infections: confusion and inconsistency in the absence of evidence or consensus. J Antimicrob Chemother. 2005;56(6):996-999.

14. Calligaro KD, Veith FJ, Schwartz ML, Dougherty MJ, DeLaurentis DA. Differences in early versus late extracavitary arterial graft infection. J Vasc Surg. 1995;22(6):680-688.

15. Bélair M, Soulez G, Oliva VL, et al. Aortic graft infection: the value of percutaneous drainage. AJR Am J Roentgenol. 1998;171(1):119-124.

16. Morris GE, Friend PJ, Vassallo DJ, Farrington M, Leapman S, Quick CR. Antibiotic irrigation and conservative surgery for major aortic graft infection. J Vasc Surg. 1994;20(1):88-95.

17. Calligaro KD, Veith FJ, Yuan JG, Gargiulo NJ, Dougherty MJ. Intra-abdominal aortic graft infection: complete or partial graft preservation in patients at very high risk. J Vasc Surg. 2003;38(6):1199-1205.

18. Numan F, Gulsen F, Solak S, Cantasdemir M. Management of endograft infections. J Cardiovasc Surg (Torino). 2011;52(2):205-223.

19. Li JS, Sexton DJ, Mick N, et al. Proposed modifications to the Duke criteria for the diagnosis of infective endocarditis. Clin Infect Dis. 2000;30(4):633-638.
International Journal of General Medicine

\section{Publish your work in this journal}

The International Journal of General Medicine is an international, peer-reviewed open-access journal that focuses on general and internal medicine, pathogenesis, epidemiology, diagnosis, monitoring and treatment protocols. The journal is characterized by the rapid reporting of reviews, original research and clinical studies across all disease areas.

\section{Dovepress}

A key focus is the elucidation of disease processes and management protocols resulting in improved outcomes for the patient.The manuscript management system is completely online and includes a very quick and fair peer-review system. Visit http://www.dovepress.com/ testimonials.php to read real quotes from published authors. 